\section{RICYDE. Revista Internacional de Ciencias del Deporte} doi: $10.5232 /$ ricyde

Rev. int. cienc. deporte

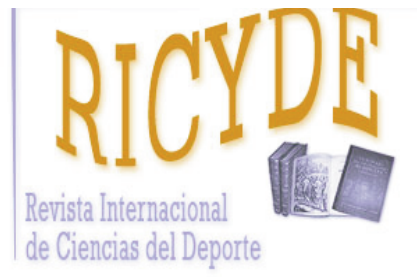

RICYDE. Revista Internacional de Ciencias del Deporte VOLUMEN XIII - AÑO XIII

Paginas:370-380 ISSN:1885-3137

Número 50 - Octubre - 2017

\title{
Efectos de un programa basado en juegos reducidos sobre la condición física de jóvenes jugadores de fútbol \\ Effects of a program based on small-sided games on the physical condition in young football players
}

\author{
Pedro Arrieta1, Julen Castellano1, Ibai Guridi1,2, Ibon Echeazarra1,2 \\ 1. Universidad del País Vasco. España \\ 2. Deportivo Alavés S.A.D. España
}

\section{Resumen}

La metodología de entrenamiento en fútbol ha evolucionado en gran medida en los últimos años, incluyendo tareas cada vez más específicas, y adquiriendo los juegos reducidos (JR) un protagonismo destacado. EI objetivo del presente estudio fue analizar los efectos sobre la condición física de jóvenes futbolistas de un programa de seis semanas basado en JR. Participaron 22 futbolistas de dos grupos de edad diferente: U16 ( $n=11$, edad: $15,8 \pm 0,3$ años) y $\cup 18(n=11$, edad: $18,5 \pm 0,5$ años). Los jugadores fueron evaluados tanto al principio como al final de la intervención con las siguientes pruebas: salto con contramovimiento (CMJ), test de velocidad en $40 \mathrm{~m}$ (V40m) y test de resistencia University of Montreal Track Test (UMTT). Los resultados mostraron mejoras significativas del pre- al post- test en el CMJ (ES $=1,05 \pm 0,13,100 \%, 0 \%$, $0 \%), \mathrm{V} 40 \mathrm{~m}(\mathrm{ES}=-0,29 \pm 0,15,0 \%, 16 \%, 84 \%)$ y UMTT $(\mathrm{ES}=0,30 \pm 0,20,79 \%, 21 \%, 0 \%) \mathrm{p}<0,05$. Con dichos resultados podemos concluir que un programa basado en JR, con una secuencia progresiva y con variedad en los formatos de juego en cuanto a dimensiones y número de jugadores por equipo, puede mantener y/o mejorar la condición física de los futbolistas. Una de las aplicaciones prácticas derivada del estudio es que los entrenadores pueden implementar JR para entrenar mediante tareas jugadas sin descuidar el aspecto condicional de los jugadores.

Palabras clave: football; small-sided games; physical condition; test.

\begin{abstract}
Football training methodology has evolved greatly in recent years, including more increasingly specialized drills, with small-side games (SSG) taking on a prominent role. The objective of the current study was to analyze the effects that SSGs have on the physical condition of young football players during a six-week program. The study included 22 football players of two different age groups: U16 ( $n=11$, age: $15.8 \pm 0.3$ years) and $U 18(n=11$, age: $18.5 \pm 0.5$ years). The participants were evaluated at the beginning and at the end of the intervention with the following tests: counter-movement jump (CMJ), $40 \mathrm{~m}$ sprint speed test (V40m) and endurance test "University of Montreal Track Test (UMTT)". The results showed significant improvements of the pre- to post- test on the CMJ $(E S=1.05 \pm 0.13,100 \%, 0 \%, 0 \%), \mathrm{V} 40 \mathrm{~m}(\mathrm{ES}=0.29 \pm$ $0.15,0 \%, 16 \%, 84 \%)$ and UMTT (ES $=0.30 \pm 0.20,79 \%, 21 \%, 0 \%) p<0.05)$. With these results we can conclude that a program based on SSG, as well as progressive sequence and variety in the game formats in terms of the size and number of players on a team, can maintain and/or improve the physical condition of the players. One of the practical applications derived from the study is that the coaches can implement SSG to develop game tasks without neglecting the players' physical condition.
\end{abstract}

Key words: football; small-sided games; physical condition; test. 
Arrieta, P.; Castellano, J.; Guridi, I., y Echeazarra, I. (2017). Efectos de un programa basado en juegos reducidos sobre la condición física de jóvenes jugadores de fútbol. RICYDE. Revista internacional de ciencias del deporte, 50(13), 370-380. https://doi.org/10.5232/ricyde2017.05004

\section{Introducción}

L a metodología de entrenamiento en fútbol ha ido evolucionando en gran medida en los últimos años (Clemente, Martins, y Mendes, 2014a; Nevado-Garrosa y Suárez-Arrones, 2015). Con el fin de realizar entrenamientos cada vez más específicos surgen los entrenamientos basados en JR (Casamichana, San Román, Calleja, y Castellano, 2015; Clemente y col., 2014a; Dellal, Varliette, Owen, Chirico, y Pialoux, 2012; Little, 2009), pudiéndose periodizar (Castellano y Casamichana, 2016) a lo largo del proceso de entrenamiento. Este tipo de tareas incluidas en el mismo dominio de acción motriz (Parlebas, 2001) parecen garantizar la transferencia en el aprendizaje. Del mismo modo, son adaptadas y propuestas por el cuerpo técnico en función de sus objetivos formativos (Clemente, Martins, y Mendes, 2014b; Nevado-Garrosa y col., 2015). La importancia de la intervención a partir de este tipo de tareas es que reproducen las demandas de la competición (NevadoGarrosa y col., 2015), permitiendo estimular de manera global la condición física y técnicotáctica (Clemente y col, 2014b; Martín-Martínez, Reigal-Garrido, Chirosa-Ríos, HernándezMendo, Chirosa-Ríos, Martin-Tamayo, y Guisado-Barrilao, 2015; Nevado-Garrosa y col., 2015) de los jugadores y equipos.

En los últimos años, gran cantidad de investigaciones se han centrado en analizar los efectos agudos (Clemente y col., 2014a, 2014b; Faude, Steffen, Kellmann, y Meyer, 2014; Hill-Hass, Coutts, Rowsell, y Dawson, 2009; Impellizzeri, Marcora, Castagna, Reilly, Sassi, Iaia, y Rampinini, 2006) que tiene la intervención en el proceso de entrenamiento a partir de JR sobre la condición física. Las respuestas agudas dependen en gran medida de la modificación de ciertas variables estructurales como: las dimensiones del espacio (Casamichana, San Román, Calleja, y Castellano, 2015; Casamichana y Castellano, 2010; Casamichana, Castellano, y Dellal, 2012), exigiendo una mayor demanda a medida que las dimensiones se incrementan; el número de jugadores (Casamichana y col., 2012, 2015), aumentando la exigencia fisiológica cuando el número es reducido; duración de la tarea (Casamichana y col., 2015; Clemente y col., 2014a) y de las pausas (Casamichana y col., 2015; Clemente y col., 2014a), aumentando la demanda fisiológica cuando las tareas se alargan y reducen sus descansos; el número de repeticiones (Casamichana y col., 2015; Clemente y col., 2014a), aumentando las demandas fisiológicas cuando éstas se incrementan; inclusión de comodines (Casamichana y col., 2012, 2015), reduciendo las demandas fisiológicas para el equipo en superioridad, o; el aliento del entrenador (Rampinini, Impellizzeri, Castagna, Abt, Chamari, Sassi, y Marcora, 2007), que hacen aumentar las demandas cuando el entrenador está presente, entre otros factores (Clemente y col., 2014a). Sin embargo todavía existe poca literatura en relación a si estos efectos agudos pueden convertirse en efectos crónicos, es decir, puedan ser sustitutivos de otro tipo de actividades más analíticas para obtener cambios en la condición física a medio o largo plazo.

Aunque el estudio de los efectos crónicos a partir de los JR no ha sido tan atendido como para analizar los efectos agudos, su interés se ha visto incrementado (Buchheit, Simpson, Peltola, y Méndez-Villanueva, A, 2009; Dellal y col., 2012; Faude y col., 2014; Iacono y col., 2015; Impellizzeri y col., 2006; Jastrzebski y col., 2014; Kelly, Gregson, Reilly, y Drust, 2013; Iacono, Eliakim, y Meckel, 2015; Los Arcos, Vázquez, Martín, Lerga, Sánchez, Villagra, y Zulueta,, 2015; Radziminski, Rompa, Barnat, Dargiewicz, y Jastrzebski, 2013; Reilly y White, 2004). Así por ejemplo, se ha estudiado si existen diferencias entre intervenir con JR respecto a realizar tareas interválicas de alta intensidad o High Intensity Interval Training (Buchheit y col., 2009; Dellal y col., 2012; Faude y col., 2014;Iacono y col., 2015; Impellizzeri y col., 2006; Jastrzebski, Barnat, Dargiewicz, Jaskulska, Szwarc, y Radziminski, L., 2014; Kelly y col., 2013; Los Arcos y col., 2015; Radziminski y col., 2013; Reilly y col., 
Arrieta, P.; Castellano, J.; Guridi, I., y Echeazarra, I. (2017). Efectos de un programa basado en juegos reducidos sobre la condición física de jóvenes jugadores de fútbol. RICYDE. Revista internacional de ciencias del deporte, 50(13), 370-380. https://doi.org/10.5232/ricyde2017.05004

2004), o actividades relativas a la habilidad por repetir esprines o Repeated Sprint Ability (Hill-Hass y col., 2009). La mayoría de las investigaciones coinciden en que metodologías basadas en JR podrían ser igualmente efectivas para la mejora del rendimiento físico (Buchheit y col., 2009; Dellal y col., 2012; Faude y col., 2014; Iacono y col., 2015; Impellizzeri y col., 2006; Jastrzebski y col., 2014), con la ventaja añadida de que las intervenciones basadas en JR ofrecen a los jugadores una mayor especificidad e integración de aspectos físicos, técnico-tácticos y decisionales (Casamichana y col., 2015).

A pesar de la probable efectividad crónica de los JR (Hill-Hass y col., 2009; Owen, Wong, Paul, y Dellal, 2012), algunos trabajos señalan un posible efecto techo o ceiling effect, bien por una alta capacidad física (Faude y col., 2014; Hill-Haas, Dawson, Impellizzeri, y Coutts, 2011; Hoff, Wisløff, Engen, Kemi, y Helgerud, 2002) o calidad (Stevens, De Ruiter, Beek, y Savelsbergh, 2015) de los jugadores. En dichos trabajos se argumenta que los jugadores no son estimulados lo suficiente y a pesar de una implementación prolongada de los mismos formatos y prescripción (duraciones y repeticiones) de JR, la condición física podría no verse mejorada (Impellizzeri y col., 2006). Sin embargo surge la duda de si la metodología seguida por los estudios anteriormente citados, donde no se contempló una progresión gradual en la prescripción de los JR (Hill-Hass y col., 2009; Owen y col., 2012) o la intervención no fue prolongada en el tiempo (Owen y col., 2012), pudiera haber influido en los resultados obtenidos, en el sentido de que dichas intervenciones no fueran lo suficientemente estimulantes para los jugadores.

Por todo lo anterior, el objetivo del presente trabajo fue analizar los efectos crónicos sobre la condición física de jóvenes futbolistas mediante un programa de intervención basado en JR implementado durante 6 semanas. En la intervención tanto los formatos de los JR como la prescripción (número de series y repeticiones, y duración de las repeticiones y pausas) siguieron una secuencia preestablecida en progresión. Los resultados del presente trabajo podrían ayudar a entrenadores y preparadores físicos a implementar tareas jugadas más próximas a los rasgos del fútbol, con las que poder desarrollar su 'modelo de juego', sin descuidar el aspecto condicional de los jugadores.

\section{Participantes \\ Método}

En el presente estudio tomaron parte 38 jugadores de dos categorías inferiores de un club profesional de la liga española, aunque únicamente se tuvieron en cuenta para la valoración final 22 jugadores, los cuales completaron más del $80 \%$ del entrenamiento y de la competición, así como la realización de las valoraciones pre y post intervención: jugadores cadete (U16, $\mathrm{n}=11$, edad: $15,3 \pm 0,4$ años, altura: $171,9 \pm 6,8 \mathrm{~cm}$, peso: $67,1 \pm 5,2 \mathrm{~kg}, \mathrm{~V}_{\max }$ : $26,1 \pm 3,1 \mathrm{~km} \cdot \mathrm{h}^{-1}$ ) y jugadores juveniles (U18, $\mathrm{n}=11$, edad: $18,2 \pm 0,5$ años, altura: $179,1 \pm 3,5$ cm, peso: $71,3 \pm 4,3 \mathrm{~kg}, \mathrm{~V}_{\text {max }}: 26,8 \pm 2,9 \mathrm{~km} \cdot \mathrm{h}^{-1}$ ). Todos los jugadores tenían al menos cinco años de experiencia en la práctica de fútbol (siete en el caso de los juveniles), y realizaban al menos tres sesiones de entrenamiento $(3 * 90 \mathrm{~min})$ y un partido de competición $(80$ min para cadetes y 90 min para juveniles) a la semana.

Todos los jugadores, padres-madres y/o tutores, junto a los responsables del club, fueron notificados del diseño de la investigación y sus requisitos, así como de los beneficios y riesgos potenciales antes de iniciar el estudio. Todos firmaron el consentimiento informado. El trabajo está diseñado de acuerdo a las condiciones éticas de la Declaración de Helsinki. La Comisión de Ética de la Universidad del País Vasco aprobó el estudio. 
Arrieta, P.; Castellano, J.; Guridi, I., y Echeazarra, I. (2017). Efectos de un programa basado en juegos reducidos sobre la condición física de jóvenes jugadores de fútbol. RICYDE. Revista internacional de ciencias del deporte, 50(13), 370-380. https://doi.org/10.5232/ricyde2017.05004

\section{Intervención con small-sided games}

El programa de intervención consistió en implementar distintos formatos de JR a lo largo de seis semanas en las sesiones habituales de entrenamiento. Durante el tiempo que duró la intervención, los jugadores realizaron una media de tres sesiones de entrenamiento a la semana, en dos de las cuales realizaron un tipo de JR prescrito. La propuesta de JR se basó en ir implementando de manera progresiva distintos $\mathrm{JR}$, variando el número de jugadores por equipo (con presencia o no de comodines), dimensiones de terreno de juego, número de repeticiones y de series, duración de cada repetición y tiempos de descanso entre repeticiones y series (Tabla 1). En todos los JR prescritos se pidió a los jugadores la máxima continuidad en el juego, evitando parones o pérdidas de tiempo durante las tareas (Casamichana y col., 2012). Con el objetivo de no reducir el ritmo del partido hubo numerosos balones disponibles, de manera que cada vez que uno salía fuera, otro era rápidamente introducido en el juego desde una de las porterías. Los entrenadores estuvieron presentes dando aliento a los jugadores para mantener el ritmo de juego durante todos los JR (Rampinini y col., 2007).

Tabla 1. Formatos y prescripción de los JR en las seis semanas de intervención. Semana: número de la semana de la intervención. Jugadores por equipo: número de jugadores en cada equipo. Espacio relativo: metros cuadrados por jugador en $\mathrm{m}^{2}$. Rep: número de repeticiones. Dur: duración de cada repetición. Pausa/s: Pausa entre series. Pausa/rep: pausa entre repeticiones.

\begin{tabular}{|c|c|c|c|c|c|c|c|}
\hline Semana & $\begin{array}{l}\text { Jugadores } \\
\text { por equipo }\end{array}$ & $\begin{array}{l}\text { Espacio relativo } \\
\left(\mathrm{m}^{2} / \mathrm{jug}\right)\end{array}$ & $\begin{array}{l}\text { Serie } \\
(\mathrm{n})\end{array}$ & $\begin{array}{l}\text { Rep } \\
\text { (n) }\end{array}$ & $\begin{array}{l}\text { Dur } \\
(\min )\end{array}$ & $\begin{array}{l}\text { Pausa/s } \\
\text { (min) }\end{array}$ & $\begin{array}{l}\text { Pausa/rep } \\
\quad \text { (min) }\end{array}$ \\
\hline \multirow{2}{*}{$1^{\mathrm{a}}$} & 4 & 100 & 1 & 6 & 2 & 0 & 1 \\
\hline & 8 & 200 & 1 & 4 & 9 & 0 & 2 \\
\hline \multirow{2}{*}{$2^{a}$} & 4 & 100 & 1 & 6 & 2,5 & 0 & 1 \\
\hline & 8 & 200 & 1 & 4 & 10 & 0 & 2 \\
\hline \multirow{2}{*}{$3^{\mathrm{a}}$} & 2 & 50 & 2 & 3 & 2 & 0 & 1 \\
\hline & 4 & 150 & 1 & 6 & 4,5 & 0 & 2 \\
\hline \multirow{2}{*}{$4^{\mathrm{a}}$} & 2 & 50 & 2 & 4 & 2 & 0 & 1 \\
\hline & 4 & 150 & 1 & 6 & 5 & 0 & 2 \\
\hline \multirow{2}{*}{$5^{\mathrm{a}}$} & 8 & 150 & 1 & 3 & 6 & 0 & 1 \\
\hline & 2 & 100 & 2 & 6 & 2 & 1,5 & 1 \\
\hline \multirow{2}{*}{$6^{\mathrm{a}}$} & 8 & 150 & 1 & 3 & 6,5 & 0 & 1 \\
\hline & 2 & 100 & 2 & 6 & 2 & 1 & 1 \\
\hline
\end{tabular}

\section{Test físicos}

Para medir la potencia del tren inferior se realizó el test de salto con contramovimiento (CMJ) siguiendo el procedimiento de estudios anteriores (Yanci y Los Arcos, 2014), con dos repeticiones cada jugador y un descanso de aproximadamente 15 seg entre repeticiones. Todos los jugadores realizaron los saltos en la misma plataforma de contacto (Optojump, Microgate $^{\mathrm{TM}}$, Bolzano, Italy), ya utilizada en estudios anteriores (Los Arcos y col., 2015). 
Arrieta, P.; Castellano, J.; Guridi, I., y Echeazarra, I. (2017). Efectos de un programa basado en juegos reducidos sobre la condición física de jóvenes jugadores de fútbol. RICYDE. Revista internacional de ciencias del deporte, 50(13), 370-380. https://doi.org/10.5232/ricyde2017.05004

La valoración de la velocidad y aceleración se realizó a partir de la medición de un sprint de $40 \mathrm{~m}$, distancia que parece ser suficiente para que los jugadores puedan conseguir la velocidad pico (Buchheit, Simpson, Peltola, y Méndez-Villanueva, 2012). Los jugadores comenzaron la carrera 0,5 m detrás de la primera célula fotoeléctrica (Polifemo Radio Light; Microgate $^{\mathrm{TM}}$, Bolzano, Italy), tomándose además los tiempos de paso en las distancias de 5, 10,20 y $30 \mathrm{~m}$. Esto dio como resultado la medición de las siguientes variables: $\mathrm{V}_{0-5 \mathrm{~m}}, \mathrm{~V}_{5-10 \mathrm{~m}}$, $\mathrm{V}_{10-20 \mathrm{~m}}, \mathrm{~V}_{20-30 \mathrm{~m}}, \mathrm{~V}_{30-40 \mathrm{~m}}$ y $\mathrm{V}_{0-40 \mathrm{~m}}$. El Coeficiente de Variación $(\mathrm{CV})$ de cada una de las pruebas de CMJ y velocidad, y en cada uno de los días de los test (pre y post) fue entre $0 \mathrm{y}$ $2,8 \%$, siendo los valores inferiores al $10 \%$, valor sugerido para considerar los datos fiables (Atkinson y Nevill, 1998). Finalmente, la resistencia fue valorada a partir de la prueba UMTT (Leger y Boucher, 1980) realizada en un campo de fútbol de $102 * 62 \mathrm{~m}$ y estableciendo marcas cada $50 \mathrm{~m}$. El UMTT es un test de campo de carrera incremental y máximo. Para asegurar que los jugadores mantuvieran estable su velocidad de desplazamiento en cada uno de los periodos, se señalaron los tiempos de paso cada 50 metros. La velocidad inicial de carrera del test fue de $8,5 \mathrm{~km} \cdot \mathrm{h}^{-1}$, que fue incrementando $1 \mathrm{~km} \cdot \mathrm{h}^{-1}$ cada $2 \mathrm{~min}$. Cuando el jugador no mantuvo el ritmo en dos marcas consecutivas se consideró que había terminado la prueba. El test UMTT ya ha sido validado con anterioridad en jugadores junior de fútbol (Llodio, García-Tabar, Sánchez-Medina, Ibáñez, y Gorostiaga, 2015).

\section{Procedimiento}

Tanto al inicio como al final de la intervención que se realizó durante los meses de enero y febrero de 2016, los jugadores completaron tres test (CMJ, velocidad en 40m y UMTT). Previamente los jugadores fueron informados de la forma correcta de realización de cada una de las pruebas aunque ya estaban familiarizados con las mismas. Antes de iniciar cada uno de los test, los jugadores realizaron un calentamiento específico de unos 10 min de duración. Tanto en el pre como en el post, los test fueron realizados el mismo día, en la misma franja horaria y siguiendo el mismo protocolo. En primer lugar se realizó el CMJ en un gimnasio en condiciones estables y posteriormente el test de velocidad 40m y el UMTT en un campo de fútbol de césped artificial con las condiciones ambientales propias de la zona y la época del año. Antes de la realización de los test se aseguró que los jugadores hubiesen tenido un mínimo de $48 \mathrm{~h}$ de descanso, siguiendo la recomendación de trabajos anteriores (Tulppo, Mäkikallio, Seppänen, Laukkanen, y Huikuri, 1998), para evitar signos de fatiga y obtener el máximo rendimiento en cada una de las pruebas.

Durante el tiempo que duró la intervención, los jugadores combinaron la prescripción de JR con sus rutinas habituales de entrenamiento, que incluyeron trabajos de prevención así como tareas técnico-tácticas o de estrategia, no implementándose ninguna tarea con objetivo condicional realizada de manera específica. Se determinó que los JR prescritos en la intervención formaran parte integrada de la sesión de entrenamiento, respetándose la condición que de dicha intervención ocupase la primera parte de la sesión. Los entrenamientos tuvieron una duración efectiva media de $69 \mathrm{~min}( \pm 15 \mathrm{~min})$ de manera que en los días en los que se propuso la intervención, los contenidos del mismo tuvieron una duración aproximada del 50\% de la duración total de la sesión.

\section{Análisis de datos}

Los resultados son presentados como media y desviación estándar $( \pm \mathrm{SD})$. La fiabilidad de las diferentes pruebas se calculó a partir del coeficiente de variación (Atkinson y col., 1998). Para analizar los efectos del programa de intervención se compararon los valores obtenidos 
Arrieta, P.; Castellano, J.; Guridi, I., y Echeazarra, I. (2017). Efectos de un programa basado en juegos reducidos sobre la condición física de jóvenes jugadores de fútbol. RICYDE. Revista internacional de ciencias del deporte, 50(13), 370-380. https://doi.org/10.5232/ricyde2017.05004

por los jugadores en el pre- y post- test a través de la prueba no paramétrica de Wilcoxon. Además, el tamaño del efecto se calculó utilizando la propuesta de Cohen (Cohen, 1998). Los rangos para valorar el tamaño del efecto (ES) fueron: $<0,2 ; 0,2-0,6 ; 0,6-1,2 ; 1,2-2,0 ; 2,0$ 4,0 y 2,0-4,0, considerándose como trivial, pequeño, moderado, grande, y muy grande, respectivamente (Hopkins, Marshall, Batterham, y Hanin, 2009). Los cambios cuantitativos de las diferencias fueron evaluados de forma cualitativa siguiendo la siguiente escala de interpretación de probabilidades: $<1 \%$ casi seguro que no; $1-5 \%$ muy improbable, $5-25 \%$ improbable, $25-75 \%$ no está claro, $75-95 \%$ es probable, $95-99 \%$ muy probable y, $>99 \%$ casi seguro. Las diferencias se consideraron significativas al nivel de $p<0,05$. Para el análisis de datos se utilizó el programa SSPS versión 22 para Windows, (SPSS Inc., Chicago, IL, USA) y la hoja de cálculo estadístico de Excel específica (Hopkins, 2007).

\section{Resultados}

En la Tabla 2 se recogen los valores medios y las SD del pre y post en las diferentes pruebas. A efectos prácticos, hubo mejoras en prácticamente todas las variables, aunque únicamente existieron diferencias significativas en las variables $C M J, V_{5-10 m}, V_{20-30 m}, V_{40 m}$ y UMTT.

Tabla 2. Coeficiente de variación para cada una de las pruebas. CMJ: Altura alcanzada en cm; $\mathrm{V}_{0-5 \mathrm{~m}}$ : tiempo en seg de 0 a $5 \mathrm{~m} ; \mathrm{V}_{5-10 \mathrm{~m}}$ : tiempo de 5 a $10 \mathrm{~m} ; \mathrm{V}_{10-20 \mathrm{~m}}$ : tiempo de 10 a $20 \mathrm{~m} ; \mathrm{V}_{20-30 \mathrm{~m}}$ : tiempo de 20 a $30 \mathrm{~m} ; \mathrm{V}_{30-40 \mathrm{~m}}$ : tiempo de 30 a 40m; $V_{40 m}$ : tiempo en realizar los 40m; UMTT: tiempo total en test UMTT en min.

\begin{tabular}{cccccc}
\hline & \multicolumn{2}{c}{ PreTest } & \multicolumn{3}{c}{ PosTest } \\
Pruebas & Media & \pm SD & Media & \pm SD & $\%$ dif \\
\hline CMJ $(\mathrm{cm})^{*}$ & 33,57 & 0,82 & 37,14 & 0,95 & $9,6 \%$ \\
$\mathrm{~V}_{0-5 \mathrm{~m}}(\mathrm{seg})$ & 1,04 & 0,02 & 1,04 & 0,03 & $-0,1 \%$ \\
$\mathrm{~V}_{5-10 \mathrm{~m}}(\mathrm{seg})^{*}$ & 0,76 & 0,01 & 0,75 & 0,02 & $-2,3 \%$ \\
$\mathrm{~V}_{10-20 \mathrm{~m}}(\mathrm{seg})$ & 1,30 & 0,01 & 1,29 & 0,02 & $-0,6 \%$ \\
$\mathrm{~V}_{20-30 \mathrm{~m}}(\mathrm{seg})^{*}$ & 1,24 & 0,02 & 1,21 & 0,02 & $-2,5 \%$ \\
$\mathrm{~V}_{30-40 \mathrm{~m}}(\mathrm{seg})$ & 1,21 & 0,02 & 1,21 & 0,02 & $0,0 \%$ \\
$\mathrm{~V}_{40 \mathrm{~m}}(\mathrm{seg})^{*}$ & 5,54 & 0,01 & 5,50 & 0,04 & $-0,7 \%$ \\
UMTT $(\mathrm{min}) *^{20,4}$ & 1,9 & 20,98 & 1,75 & $2,7 \%$ \\
\hline
\end{tabular}

$* p<0,05$

Las mejoras en CMJ, una vez implementado el programa de intervención, tuvieron un tamaño del efecto grande $(E S=1,05 \pm 0,13)$. La interpretación cualitativa indica que es casi seguro $(100 \%)$ que la intervención con JR tenga efectos positivos.

Con respecto a las mejoras producidas tras la intervención en la variable velocidad, cabe señalar que tuvo efectos positivos en prácticamente todas las valoraciones de la velocidad, aunque en distinta proporción, como se presenta en la Tabla 2. Los efectos más grandes los vemos en los valores de $\mathrm{V}_{20-30 \mathrm{~m}}$ donde el efecto es moderado $(-0,63 \pm 0,28)$. Seguidamente, el efecto es pequeño para $\mathrm{V}_{5-10 \mathrm{~m}}(-0,50 \pm 0,38), \mathrm{V}_{10-20 \mathrm{~m}}(-0,23 \pm 0,26)$ y $\mathrm{V}_{40 \mathrm{~m}}(-0,29 \pm 0,15)$. Por último, el efecto fue trivial o nulo para $\mathrm{V}_{0-5 \mathrm{~m}}(-0,15 \pm 0,31)$ y $\mathrm{V}_{30-40 \mathrm{~m}}(-0,01 \pm 0,15)$. La interpretación cualitativa para cada valor viene expresada en la Figura 1. 
Arrieta, P.; Castellano, J.; Guridi, I., y Echeazarra, I. (2017). Efectos de un programa basado en juegos reducidos sobre la condición física de jóvenes jugadores de fútbol. RICYDE. Revista internacional de ciencias del deporte, 50(13), 370-380. https://doi.org/10.5232/ricyde2017.05004

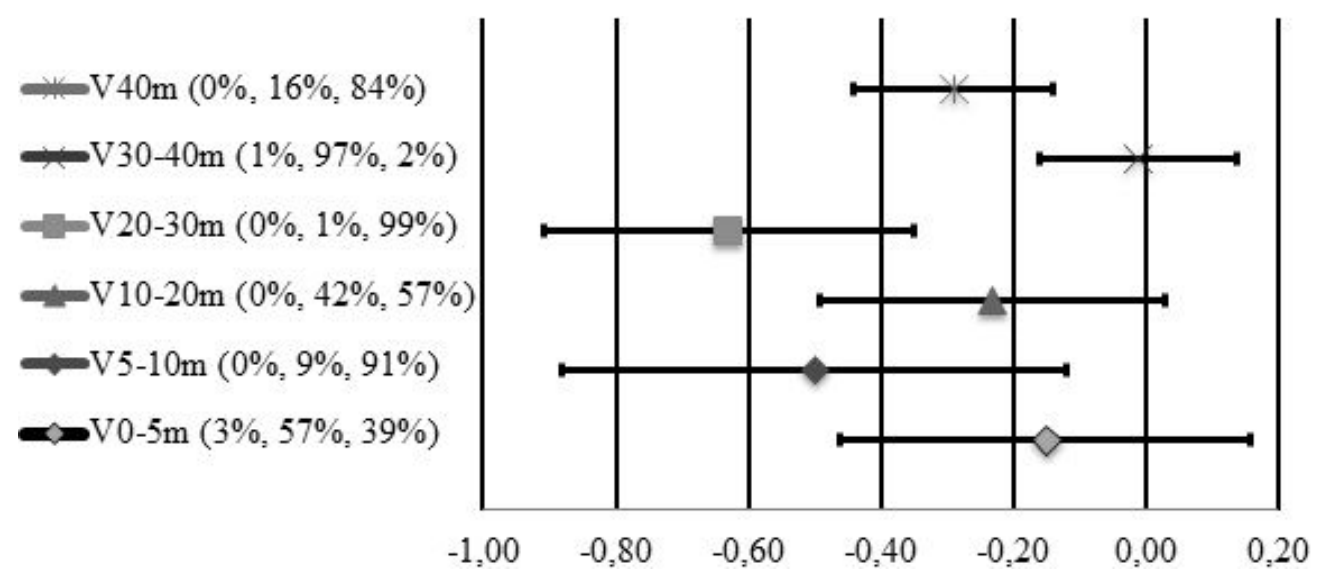

Figura 1. Los valores del tamaño del efecto para cada una de las variables de velocidad así como la interpretación cualitativa. $\mathrm{V}_{0-5 \mathrm{~m}}$ : Tiempo en seg de 0 a 5m; $\mathrm{V}_{5-10 \mathrm{~m}}$ : Tiempo de 5 a $10 \mathrm{~m} ; \mathrm{V}_{10-20 \mathrm{~m}}$ : Tiempo de 10 a 20m; $\mathrm{V}_{20-30 \mathrm{~m}}$ : Tiempo de 20 a 30m; $\mathrm{V}_{30-40 \mathrm{~m}}$ : Tiempo de 30 a 40m; $\mathrm{V}_{40 \mathrm{~m}}$ : Tiempo en realizar $\operatorname{los} 40 \mathrm{~m}$.

Finalmente, aunque existieron diferencias significativas respecto al pre $(\mathrm{p}<0,05)$ en la prueba UMTT, el efecto fue pequeño $(E S=0,30 \pm 0,20)$. La interpretación cualitativa indica que es probable (79\%) que la intervención con JR tenga efectos positivos, improbable (21\%) que no tenga ningún efecto y casi seguro $(0 \%)$ que no tenga ningún efecto la intervención.

\section{Discusión}

El objetivo del presente trabajo fue analizar los efectos en la condición física en jóvenes futbolistas a partir de la implementación de un programa de intervención de seis semanas de duración basado en JR, llevado a cabo en el periodo competitivo. A partir de las referencias consultadas, este es el primer estudio donde tanto los formatos (número de jugadores y dimensiones del terreno de juego) como la prescripción (repeticiones y duraciones) siguieron una secuencia de progresión preestablecida. El principal resultado del estudio indica que un programa basado en JR podría mejorar la condición física de los futbolistas.

A diferencia de lo aportado por estudios anteriores, donde se encontró que la intervención a partir de JR en jugadores con una condición física óptima (Faude y col., 2014; Hill-Haas y col., 2011; Hoff y col., 2002) o alta calidad de juego (Stevens y col., 2015) no permitía mejorar su rendimiento físico, probablemente debido al ceiling effect (Stevens y col., 2015), en el presente estudio, después de seis semanas de intervención, los jugadores manifestaron valores significativamente superiores en casi todas las variables físicas analizadas. Quizás esto pudo deberse a que a diferencia de lo propuesto en los estudios mencionados (Faude y col., 2014; Hill-Haas y col., 2011; Hoff y col., 2002), la intervención con JR implementada en este trabajo siguió una secuencia preestablecida y prolongada en el tiempo, así como una variedad de formatos de las tareas, concretamente las referidas al número de jugadores y las dimensiones del terreno de los JR donde los jugadores intervinieron.

Por otro lado, al igual que ocurrió en un estudio anterior (Iacono y col., 2015), tras la intervención con JR hubo mejoras en los valores de CMJ y velocidad en 40m. Algunos trabajos anteriores (Jastrzebski y col., 2014; Radziminski y col., 2013; Reilly y col, 2004) encontraron que los JR pueden ser aceptables contenidos de entrenamiento para mantener las cualidades de fuerza y velocidad durante la temporada competitiva, si bien dichos resultados mostraron un tamaño del efecto trivial. Sin embargo, existe algún trabajo (Buchheit y col., 2009) que no encontró mejoras significativas en la velocidad tras un programa de intervención con JR, si bien es verdad que este estudio tenía como objetivo mejorar la 
Arrieta, P.; Castellano, J.; Guridi, I., y Echeazarra, I. (2017). Efectos de un programa basado en juegos reducidos sobre la condición física de jóvenes jugadores de fútbol. RICYDE. Revista internacional de ciencias del deporte, 50(13), 370-380. https://doi.org/10.5232/ricyde2017.05004

capacidad aeróbica y no tanto la velocidad. Es probable que durante los JR se produzcan aceleraciones y desaceleraciones (Castellano y Casamichana, 2013) que implican especialmente a la musculatura del tren inferior (en movimientos tales como saltos, cambios de dirección, aceleraciones, paradas, etc.), las cuales podrían ser una manera eficiente de mejorar la potencia muscular del tren inferior (Iacono y col., 2015). Este tipo de acciones son mayores, cuando se realizan tareas con menor número de jugadores y en espacios más pequeños, que como se ha demostrado anteriormente (Castellano, Casamichana, y Dellal, 2013), demanda de manera especial aceleraciones y desaceleraciones. Por este motivo quizás, la inclusión en la intervención de formatos de JR pequeños (dos contra dos) a lo largo de las seis semanas pudo tener que ver con mejoras a este nivel.

Por otro lado, dimensiones grandes y número de jugadores por equipo elevados demandan a los jugadores velocidades altas (picos de velocidad) o mayores distancias recorridas a alta velocidad (Casamichana y col., 2010). Los JR diseñados en la intervención (formatos de 8:8 en dimensiones superiores a $200 \mathrm{~m}^{2}$ ) pudieron favorecer la aparición de esta demanda (Gaudino, Alberti, y Iaia, 2014), por lo que pudo ser estimulada la velocidad y, por tanto, tener como consecuencia mejoras en algunas de la variables que se midieron en el test de velocidad.

Finalmente, en la línea de lo aportado por trabajos anteriores (Buchheit y col., 2009; Casamichana y col., 2010), los JR pueden ser un buen método para mejorar la resistencia, siempre y cuando se acierte con los condicionantes de la tarea (número de jugadores o dimensiones de juego) que favorezcan la estimulación de la frecuencia cardiaca (Casamichana y col., 2010). En la presente investigación, se optó por incluir formatos medios (4:4 en dimensiones de $150 \mathrm{~m}^{2}$ ) para desarrollar esta condición de manera específica. Los resultados del estudio indicaron que los jugadores mejoraron la resistencia de manera significativa, similar a lo encontrado en otros trabajos (Buchheit y col., 2009), donde también se observaron diferencias significativas en test de resistencia como el $\mathrm{V}_{\text {FIT30/15 }}$ (Buchheit y col., 2009), RSA (Owen y col., 2012) y YOYO (Hill-Hass y col., 2009) o test específicos (Jastrzebski y col., 2014; Radziminski y col., 2013), en línea al utilizado en el presente estudio, donde se implementó la prueba UMTT (Hill-Hass y col., 2009).

Una de las mayores limitaciones del estudio es no haber podido contar con un grupo control, lo cual debe ser considerado en la interpretación de los resultados. Por otro lado, cabe subrayar también el hecho de que la intervención a partir de JR fuera solo una parte del entrenamiento, pudo influir en las mejoras encontradas en los participantes. Del mismo modo, hubiera sido muy interesante haber continuado con el proceso de intervención, es decir, alargarlo en el tiempo, para ver en qué medida la prescripción del entrenamiento mediante JR durante más semanas hubiera permitido aumentar o mantener la condición física de los jugadores, ya que esta mejora podría no ser lineal (Impellizzeri y col., 2006).

\section{Conclusiones}

A partir de los resultados obtenidos, la principal conclusión del estudio parece sugerir que un programa basado en JR, que siga una secuencia progresiva y con variedad en el formato de los juegos, puede mejorar la condición física de jóvenes futbolistas. No obstante, son necesarias más investigaciones en esta línea que permitan afinar en la comprensión de cómo la intervención con JR lleva consigo efectos crónicos en la condición física de los futbolistas.

Los resultados del presente trabajo sugieren que a partir de una intervención programada de JR a lo largo de las semanas podría no descuidar el desarrollo del aspecto condicional de los jugadores. Esto permitiría que entrenadores y preparadores físicos puedan implementar tareas 
Arrieta, P.; Castellano, J.; Guridi, I., y Echeazarra, I. (2017). Efectos de un programa basado en juegos reducidos sobre la condición física de jóvenes jugadores de fútbol. RICYDE. Revista internacional de ciencias del deporte, 50(13), 370-380. https://doi.org/10.5232/ricyde2017.05004

jugadas para desarrollar su modelo de juego sin descuidar el aspecto condicional de los jugadores.

\section{Agradecimientos}

Los autores del presente trabajo quieren agradecer al Deportivo Alavés por haber accedido a participar en esta investigación y en especial a jugadores y miembros del club que tomaron parte en el mismo. Los autores indican no tener ningún conflicto de intereses en este estudio. Además, este trabajo forma parte de la investigación LA ACTIVIDAD FISICA Y EL DEPORTE COMO POTENCIADORES DEL ESTILO DE VIDA SALUDABLE: EVALUACION DEL COMPORTAMIENTO DEPORTIVO DESDE METODOLOGIAS NO INTRUSIVAS, que ha sido subvencionado por la Secretaría de Estado de Investigación, Desarrollo e Innovación del Ministerio de Economía y Competitividad [DEP2015-66069-P, MINECO/FEDER, UE], durante el trienio 2016-2018.

\section{Referencias}

Atkinson, G., \& Nevill, A. M. (1998). Statistical methods for assessing measurement error (reliability) in variables relevant to sports medicine. Sports Medicine, 26(4), 217-238. https://doi.org/10.2165/00007256-199826040-00002

Buchheit, M.; Laursen, P. B.; Kuhnle, J.; Ruch, D.; Renaud, C., \& Ahmaidi, S. (2009). Game-based training in young elite handball players. International Journal of Sports Medicine, 30(4), 251-258.

https://doi.org/10.1055/s-0028-1105943

Buchheit, M.; Simpson, B. M.; Peltola, E., \& Méndez-Villanueva, A. (2012). Assessing maximal sprinting speed in highly trained young soccer players. International Journal of Sports Physiology and Performance, 7(1), 76-78.

https://doi.org/10.1123/ijspp.7.1.76

Casamichana, D., \& Castellano, J. (2010). Time-motion, heart rate, perceptual and motor behaviour demands in small-sides soccer games: Effects of pitch size. Journal of Sports Science, 28(14), 1615-1623.

https://doi.org/10.1080/02640414.2010.521168

Casamichana, D.; Castellano, J., \& Dellal, A. (2012). Influence of different training regimes on physical and physiological demands during small-sided soccer gamess: continuous vs. intermittent format. Journal of Strength and Conditioning Research, 27(3), 690-697.

https://doi.org/10.1519/JSC.0b013e31825d99dc

Casamichana, D.; San Román, J.; Calleja, J., \& Castellano, J. (2015). Los juegos reducidos en el entrenamiento del fútbol. Barcelona: Fútbol De Libro.

Castellano, J., \& Casamichana, D. (2013). Differences in the number of accelerations between small-sided games and friendly matches in soccer. Journal of Sports Science \& Medicine, 12(1), 209-210.

Castellano, J., \& Casamichana, D. (2016). El arte de planificar en fútbol. Barcelona: Fútbol De Libro.

Clemente, F. M.; Martins, F. M. L., \& Mendes, R. S. (2014a). Developing aerobic and anaerobic fitness using small-sided soccer games. Strength and Conditioning Journal, 36(3), 76-87.

https://doi.org/10.1519/SSC.0000000000000063

Clemente, F. M.; Martins, F. M. L., \& Mendes, R. S. (2014b). Periodization based on small-sided soccer games: theoretical considerations. Strength and Conditioning Journal, 36(5), 34-43.

https://doi.org/10.1519/SSC.0000000000000067 
Arrieta, P.; Castellano, J.; Guridi, I., y Echeazarra, I. (2017). Efectos de un programa basado en juegos reducidos sobre la condición física de jóvenes jugadores de fútbol. RICYDE. Revista internacional de ciencias del deporte, 50(13), 370-380. https://doi.org/10.5232/ricyde2017.05004

Cohen, J. (1998). Statistical power analysis for the behavioral sciences. New York: LEA.

Dellal, A.; Varliette, C.; Owen, A.; Chirico, E., \& Pialoux, V. (2012). Small-sided games versus interval training in amateur soccer players: effects on the aerobic capacity and the ability to perform intermittent exercises with changes of direction. Journal of Strength and Conditioning Research, 26(10), 2712-2720. https://doi.org/10.1519/JSC.0b013e31824294c4

Faude, O.; Steffen, A.; Kellmann, M., \& Meyer, T. (2014). The effect of short-term interval training during the competitive season on physical fitness and signs of fatigue: A crossover trial in high-level youth football players. International Journal of Sports Physiology and Performance, 9(6), 936-944. https://doi.org/10.1123/ijspp.2013-0429

Gaudino, P.; Alberti, G., \& Iaia, F. M. (2014). Estimated metabolic and mechanical demands during different small-sided games in elite soccer players. Human Movement Science, 36, 123-133.

https://doi.org/10.1016/j.humov.2014.05.006

Hill-Haas, S. V.; Dawson, B.; Impellizzeri, F. M., \& Coutts, A. J. (2011). Physiology of small-sided games training in football. A systematic review, Sports Medicine, 41(3), 199-220. https://doi.org/10.2165/11539740-000000000-00000

Hill-Hass, S. V.; Coutts, A. J.; Rowsell, G. J., \& Dawson, B. T. (2009). Generic vesus small- sided game training in soccer. International Journal of Sports Medicine, 30(9), 636-642. https://doi.org/10.1055/s-0029-1220730

Hoff, J.; Wisløff, U.; Engen, L. C.; Kemi, O.J., \& Helgerud, J. (2002). Soccer specific aerobic endurance training. British Journal of Sports Medicine, 36(3), 218-21. https://doi.org/10.1136/bjsm.36.3.218

Hopkins, W. G. (2007). A spreadhseet to compare means of two groups. Sports Science, $11,22-23$.

Hopkins, W. G.; Marshall, S. W.; Batterham, A. M. \& Hanin, J. (2009). Progressive statistics for studies in sports medicine and exercise science. Medicine and Science in Sports and Exercise, 41(1), 3-13.

https://doi.org/10.1249/MSS.0b013e31818cb278

Iacono, A. D.; Eliakim, A., \& Meckel, Y. (2015). Improving fitness of elite handball players: small-sided games vs. high-intensity intermittent training. Journal of Strength and Conditioning Research, 29(3), 835-843.

https://doi.org/10.1519/JSC.0000000000000686

Impellizzeri, F. M.; Marcora, S. M.; Castagna, C.; Reilly, T.; Sassi, A.; Iaia, F. M., \& Rampinini, E. (2006). Physiological and performance effects of generic versus specific aerobic training in soccer players. International Journal of Sports Medicine, 27(6), 483-492.

https://doi.org/10.1055/s-2005-865839

Jastrzebski, Z.; Barnat, W.; Dargiewicz, R.; Jaskulska, E.; Szwarc, A., \& Radziminski, L. (2014). Effect of in-season generic and soccer-specific high-intensity interval training in young soccer players. International Journal of Sports Science \& Coaching, 9(5), 1169-1179.

https://doi.org/10.1260/1747-9541.9.5.1169

Kelly, D. M.; Gregson, W.; Reilly, T., \& Drust, B. (2013). The development of a soccerspecific training drill for elite-level players. Journal of Strength and Conditioning Research, 27(4), 938-943.

https://doi.org/10.1519/JSC.0b013e3182610b7d 
Arrieta, P.; Castellano, J.; Guridi, I., y Echeazarra, I. (2017). Efectos de un programa basado en juegos reducidos sobre la condición física de jóvenes jugadores de fútbol. RICYDE. Revista internacional de ciencias del deporte, 50(13), 370-380. https://doi.org/10.5232/ricyde2017.05004

Leger, L., \& Boucher, R. (1980). An indirect continuous running multistage field test: the universite de montreal track test. Canadian Journal of Applied Sport Sciences, 5(2), 77-84.

Little, T. (2009). Optimizing the use of soccer drills for physiological development. Strength and Conditioning Journal, 31(3), 67-74. https://doi.org/10.1519/SSC.0b013e3181a5910d

Llodio, I.; Garcia-Tabar, I.; Sánchez-Medina, L.; Ibáñez, J., \& Gorostiaga, E. M. (2015). Estimation of the maximal lactate steady state in junior soccer players. International Journal of Sports Medicine, 36(14), 1142-1148.

https://doi.org/10.1055/s-0035-1554643

Los Arcos, A., Vázquez, J. S., Martín, J., Lerga, J., Sánchez, F., Villagra, F., \& Zulueta, J. J. (2015). Effects of small-sided games vs. interval training in aerobic fitness and physical enjoyment in young elite soccer players. PLoS One, 10(9), 16-19.

https://doi.org/10.1371/journal.pone.0137224

Martín-Martínez, I.; Reigal-Garrido, R. E.; Chirosa-Ríos, L. J.; Hernández-Mendo, A.; Chirosa-Ríos, I; Martin-Tamayo, I., y Guisado-Barrilao, R. (2015). Efectos de un programa de juegos reducidos en la percepción subjetiva del esfuerzo en una muestra de chicas adolescentes. Cuadernos de Psicología del Deporte, 15(3), 89-98. https://doi.org/10.4321/S1578-84232015000300008

Nevado-Garrosa, F., y Suárez-Arrones, L. (2015). Comparación de las demandas físicas de tareas de fútbol reducido y la competición en jugadoras de fútbol sub 13. Cultura, Ciencia y Deporte, 30, 235-243.

Owen, A. L.; Wong, D. P.; Paul, D., \& Dellal, A. (2012). Effects of a periodized smallsided game training intervention on physical performance in elite professional soccer. Journal of Strength and Conditioning Research, 26(10), 2748-2754.

https://doi.org/10.1519/JSC.0b013e318242d2d1

Parlebas, P. (2001). Léxico de praxiologia motriz. Barcelona: Paidotribo.

Radziminski, L.; Rompa, P.; Barnat, W.; Dargiewicz, R., \& Jastrzebski, Z. (2013). A comparison of the physiological and technical effects of high-intensity running and small-sided games in young soccer players. International Journal of Sports Science \& Coaching, 8(3), 455-465.

https://doi.org/10.1260/1747-9541.8.3.455

Rampinini, E.; Impellizzeri, F. M.; Castagna, C.; Abt, G.; Chamari, K.; Sassi, A. \& Marcora, S. M. (2007). Factors influencing physiological responses to small-sided soccer games. Journal of Sports Science, 25, 659-666.

https://doi.org/10.1080/02640410600811858

Reilly, T., \& White, C. (2004). Small-sided games as an alternative to interval-training for soccer players. Journal of Sports Science. 22(6), 559.

Stevens, T. G. A.; De Ruiter, C. J.; Beek, P. J., \& Savelsbergh, G. J. P. (2015). Validity and reliability of 6-a-side small-sided game locomotor performance in assessing physical fitness in football players. Journal of Sports Science, 34(6), 527-534. https://doi.org/10.1080/02640414.2015.1116709

Tulppo, M. P.; Mäkikallio, T. H.; Seppänen, T.; Laukkanen, R. T., \& Huikuri, H. V. (1998). Vagal modulation of heart rate during exercise: effects of age and physical fitness. American Journal of Physiology, 274(2 Pt 2), 424-429.

https://www.ncbi.nlm.nih.gov/pubmed/9486244

Yanci, J., \& Los Arcos, A. (2014). Muscle strength and leg asymmetries in elite runners and cyclist. International SportMed Journal, 15(3), 285-297. 